\title{
Research on statistical method of channel information transmission risk probability in large-scale wireless communication system
}

\author{
MA feng ${ }^{1}$,WU Hong-e ${ }^{2}$ \\ \{mafeng3213@163.com¹,wuhonghe321313@163.com² \\ (1. School of Mathematics and Statistics,Nanyang Institute of Technology,Nanyang 473000 China; \\ 2.School of Mathematics and Statistics,Nanyang Institute of Technology,Nanyang 473000 China)
}

\begin{abstract}
In order to improve the communication security of the large-scale wireless communication system channel during the information transmission process, the probability transmission method is used to analyze the information transmission risk and provide data reference for the operation of the wireless communication system.Follow the operating principle of a large-scale wireless communication system to build a system's information transmission channel model, and install information acquisition equipment to obtain channel information. Based on this, the optimal risk decision rule is established, and the channel transmission interference is predicted. At the same time, the channel fading characteristics are analyzed.The statistical value of the risk probability is obtained by calculating the interference prediction results and the channel fading characteristics of the system information transmission channel.Through comparative experiments, it is concluded that compared with the traditional statistical methods of risk analysis, the statistical error of the risk probability obtained by the design method is reduced by about $56 \%$. Applying this method to the daily work of the system can effectively maintain the stability of wireless communication and safety.
\end{abstract}

Keywords : Wireless communication system; communication channel; information transmission; risk probability statistics;

\section{Introduction}

A wireless communication system, also called a radio communication system, is a system consisting of three parts: a transmitting device, a receiving device, and a wireless channel. It uses wireless electromagnetic waves to implement information and data transmission.According to the classification of working frequency band or transmission means, it can be divided into medium wave communication, short wave communication, ultra short wave communication, microwave communication and satellite communication [1]. The new generation mobile communication system is a stage and goal in the evolution of mobile communication systems. It not only uses new wireless transmission technologies to improve the performance of communication systems, but also integrates with existing various wired 
and wireless networks to ensure system communication. At the same time, the communication range of the system is expanded, and a large-scale wireless communication system is obtained.

The use of a large-scale wireless communication system for transmission of related data requires the use of the system's wireless communication channel.The wireless communication channel is a physical channel for transmitting information, that is, a wireless frequency band, and it is a data signal transmission channel using wireless signals as transmission media.At present, the mainstream wireless protocols are all formulated by the IEEE. In the three wireless standards recognized by the IEEE, IEEE802.11b, IEEE802.11g, and IEEE802.11a, the number of channels is different.In the case of $802.11 \mathrm{~b} / \mathrm{g}$, the available channels will overlap and interleave in frequency, resulting in only three non-overlapping channels in the service area covered by the network. As a result, users in this service area can only share the data bandwidth of these three channels. These three channels will also be interfered by other radio signal sources, because the $802.11 \mathrm{~b} / \mathrm{gWLAN}$ standard uses the most commonly used $2.4 \mathrm{GHz}$ radio frequency band.

However, the channels of large-scale wireless communication systems are susceptible to the influence of radio waves and other channels in the external environment during the transmission of information, leading to risks such as signal interruption, poor communication, and signal loss in the communication system.In order to ensure the stable reliability and security of the system operation, it is necessary to use corresponding methods to carry out risk statistics and assessment on the channels of large-scale wireless communication systems before information transmission, and refer to the statistical results to select a more secure channel for transmission to avoid transmission signal interruption. And loss, thereby ensuring system communication stability.

After a long period of research and application, it has been found that using the risk probability as the evaluation index of the system transmission channel can directly reflect the risk value of the current communication channel, thereby quickly determining the feasibility of channel information transmission. The risk probability assessment method can generally be divided into two steps, one is the identification and analysis of the risk, and the other is the calculation of the risk value.Among them, risk identification refers to identifying potential problems that may lead to cost overruns, delayed schedules, or reduced performance, and a qualitative analysis of their consequences [2].Risk assessment refers to quantifying the risks and consequences of potential problems and determining their severity.This may involve the comprehensive application of multiple models, and finally get a comprehensive impression of system risk.Risk analysis is the evaluation and estimation of risk impacts and consequences, including qualitative and quantitative analysis.By integrating the risk analysis results and 
quantifying them, the risk probability value of the corresponding communication channel can be obtained.However, the single risk assessment is accidental and its application value in actual work is low.In order to solve the above problems, a probability statistics method is introduced to realize the risk assessment of channel information transmission in a large-scale wireless communication system, and the statistical results of the risk probability are obtained.

Probability statistics is a mathematical method to study the statistical laws of random phenomena in nature.Probability theory, as a branch of mathematics, generally includes the probability of random events, statistical independence, and deeper regularity.Probability is a quantitative indicator of the likelihood of a random event.In an independent random event, if the frequency of an event in all events, it is relatively stable in a larger range around a fixed constant.It can be considered that the probability of this event occurring is this constant [3].The probability value for any event must be between 0 and 1.Combining the probability statistics method in the mathematical category, and merging it with the channel risk estimation of large-scale wireless communication systems, the transmission communication risk is quantified, and the statistical analysis results are used as a reference.Indirectly guarantee the stable and safe operation of the communication system.

\section{Design of statistical methods for information transmission risk probability}

In order to quantify the risk of channel information transmission in a large-scale wireless communication system, the risk is converted into a probability to be expressed in combination with probability statistics, and the statistical results of comprehensive risk probability are obtained by following statistical theory.The ultimate purpose of designing the system channel information transmission risk probability statistical method is to provide data references for information transmission in wireless communication systems, thereby ensuring the communication security of information data.The implementation of the channel information transmission risk probability statistics method for large-scale wireless communication systems is shown in Figure 1. 


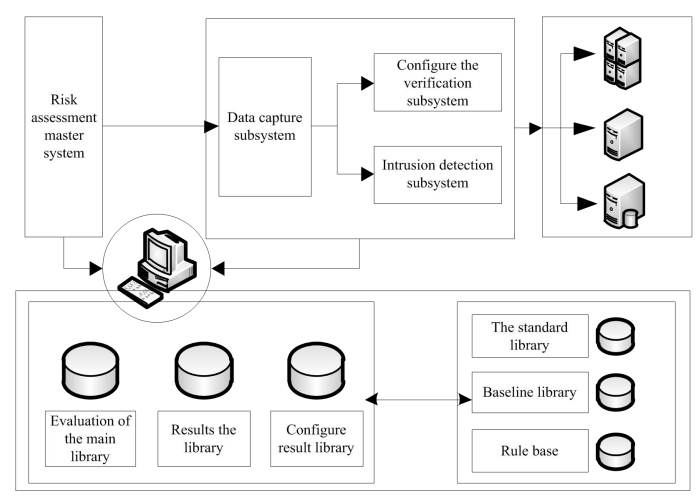

Fig. 1 Statistical block diagram of channel information transmission risk probability

\subsection{Construction of a large-scale wireless communication system transmission channel model}

There are two main channel modeling methods based on large-scale wireless communication systems: statistical channel models and deterministic channel models. The construction of the statistical model needs to be based on a large amount of actual measurement data and obtain a large number of time-domain frequency-domain channel responses through multiple measurements in different scenarios.Related channel large-scale and small-scale parameters are extracted in further statistical analysis [4].The method used for deterministic modeling is to physically reconstruct the propagated scene through computer simulation. The impulse response of the analog signal in the actual channel is calculated based on the electromagnetic wave propagation characteristics.In order to improve the application performance of the channel model, a transmission channel model of a large-scale wireless communication system is established in accordance with the flow of Figure 2 in combination with two model construction methods.

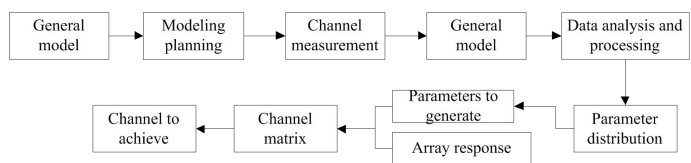

Fig. 2 Transmission channel model of large-scale wireless communication system

\subsubsection{Establish large-scale wireless communication system}

The large-scale wireless communication system must provide many important messages or services to the target node through the common channel, including synchronization signal, reference signal, control signal and multimedia broadcast multicast service.For the actual spatial correlation channel, a reasonable method is to make the transmitted signal of the system have the spatial omnidirectional characteristics, and then complete the comprehensive 
coverage.Within a certain range, the omnidirectional transmission of the system common signal is shown in Figure 3.

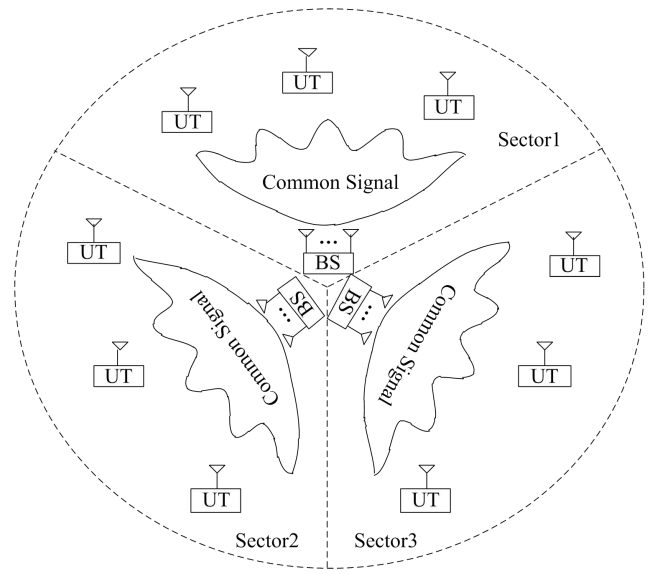

Fig. 3. Signal transmission diagram of large-scale wireless communication system

\subsubsection{Connecting communication transmission channels}

Suppose $h \in C^{M \times 1}$ represents the channel vector between the antenna array and a single antenna target node in a large-scale antenna communication system, $h \sim C N(0, R)$. For the single ring scattering model under the far-field assumption, the channel covariance matrix can be expressed as:

$$
R=\int_{-\frac{\pi}{K}}^{\frac{\pi}{K}} v(\theta)(v(\theta))^{H} p(\theta) d \theta
$$

In the formula (1), variables $v(\theta)$ and $p(\theta)$ represent the response vector and angular power spectrum of the uniform linear array respectively.Angle power spectrum $p(\theta)$ may obey different distribution according to different terrain scenes [5].

\subsubsection{Analog wireless communication transmission}

Considering the transmission scenario of large-scale wireless communication system, we focus on a subcarrier of a symbol to be transmitted.The common signal $s \in C^{M \times 1}$ is transmitted from $\mathrm{M}$ antennas of the system to all communication nodes. The signal obtained from the perspective of a communication receiving node can be expressed as:

$$
y=\sqrt{\rho} h^{H} s+z \quad(2)
$$

Formula $2 \rho$ is the total average transmission power of the communication system. The specific information transmission process is shown in Figure 4. 


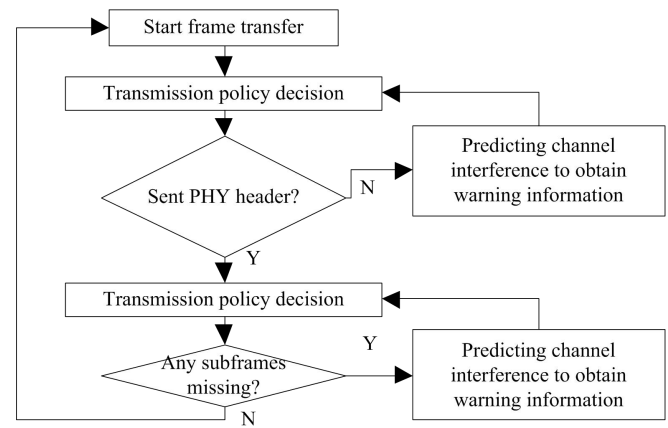

Fig. 4. Flow chart of information transmission

\subsection{Obtain system statistics channel information}

In the channel model of the large-scale wireless communication system, the channel information collection equipment is installed on the corresponding channel location, and the channel information of the cargo system is collected according to the method shown in Figure 5.

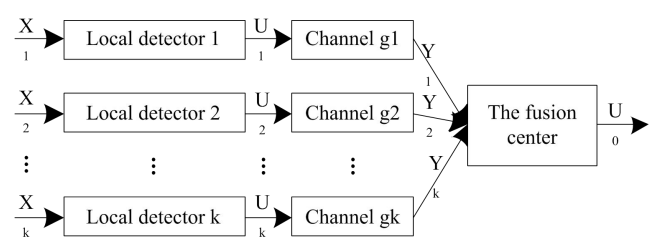

Fig. 5. Schematic diagram of information collector

Considering the uplink transmission link in beam domain of large-scale wireless communication system, $\bar{y}_{m} \in C^{T \times 1}$ represents the received signal of the m-th beam on the server side of the system, and $\bar{h}_{m} \in C^{K \times 1}$ represents the channel from all users to the m-th beam on the server side of the system, then the received signal of the m-th beam on the server side can be expressed as:

$$
\bar{y}_{m}=X \bar{h}_{m}+\bar{z}_{m}
$$

Then the received signals of all beams on the server side of the system can be expressed as:

$$
\bar{Y}=X \bar{H}+\bar{Z}
$$

The matrix corresponding to $\bar{Y}, \bar{H}$ and $\bar{Z}$ is: 
$\left\{\begin{array}{l}\bar{Y}=\left[y_{1}, \cdots, y_{m}\right] \\ \bar{H}=\left[\bar{h}_{1}, \cdots, \vec{h}_{m}\right] \\ \bar{Z}=\left[\bar{z}_{1}, \cdots, \bar{z}_{m}\right]\end{array}\right.$

Taking the communication information received by the server as the original, the channel information of the system can be obtained by using the mapping principle.

\subsection{Establish the optimal risk decision rules}

The purpose of establishing the optimal risk decision rule is to determine whether there is transmission risk in the channel based on the acquired channel transmission information of wireless communication system [6].Firstly, the input scan results and matching rules are processed to determine whether they meet the format requirements.If the format requirements are met, the scan results and matching rules are split according to the match sub result separator.

If the format requirements are not met, the corresponding error handling is called[7].Because there is a corresponding relationship between the scanning results and the matching rules, the number of disassembly results of the scanning results and the matching rules is the same, and they correspond to each other.Further processing is carried out for each scan sub result and matching item pair. The matching item type field in the matching item is used to determine the category of the matching item. The number type matching item and character type matching item correspond to different response results respectively.

\subsection{Prediction channel transmission interference}

The transmission interference prediction is carried out for the channel with transmission risk. The types and modes of channel interference are shown in Table 1:

Table 1. Channel interference factor type

\begin{tabular}{ccc}
\hline Jamming type & Missing part & Reason for frame loss \\
\hline Synchronous & PHY head & Transmission failure due to frame collision of nodes \\
jamming & & in the listening range \\
Asynchronous & PHY head、MPDU & Data transmission outside the monitoring range \\
interference & subframe & interferes with frame transmission \\
Physical interference & PHY head、MPDU & Frame damage caused by channel physical factors \\
& subframe &
\end{tabular}

For any node $i$, the probability that it is affected by synchronous interference, asynchronous interference, and physical interference and cause frame transmission failure is denoted as $P_{i}^{s y n}, P_{i}^{a s y}$, and $P_{i}^{p h y}$ respectively.Considering that physical interference has little and stable influence on frame transmission, in order to simplify interference prediction 
method, quantitative value $\phi$ is used to represent probability $P_{i}^{p h y}$ [8].Then the prediction of channel interference can be realized according to the flow shown in Fig. 6.

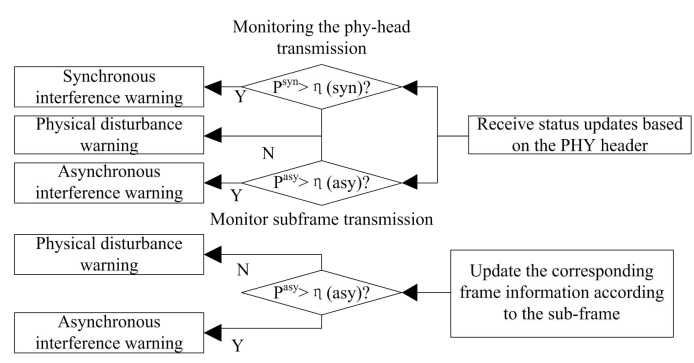

Fig. 6. Channel interference prediction flow chart

Assume that there are $N_{i}$ other sending nodes in the monitoring range of node $\mathrm{i}$, and there may be other sending nodes outside its monitoring range, and use frame transmission status information to derive the prediction results of synchronous interference and asynchronous interference.

\subsection{Analyze channel fading characteristics}

In a complex environment, the propagation path of a signal is diverse. One path may be a simple line-of-sight propagation, or it may pass through various non-visual paths through various modes such as diffuse reflection and diffraction. Distance spread.The wireless communication channel has a strong openness. At the same time, the complex receiving environment and the mobility of the receiver make the channel analysis extremely complex and time-varying [9].Based on the prediction results of channel transmission interference, the characteristics of channel fading risk are analyzed. In general, the channel fading is in a composite form, as shown in Figure 7.

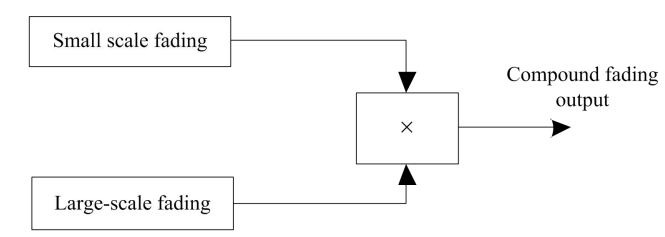

Fig. 7. Principle diagram of composite fading

It can be seen from the figure that the composite fading consists of two parts: small-scale fading and large-scale fading.Among them, path loss and shadow fading describe the intensity change of the average receiving power within a certain moving distance when the distance between the transceiver is long, which usually refers to the situation of more than dozens of wavelengths, so they are collectively called large-scale fading.However, multipath fading is 
caused by the different transmission paths, different transmission directions and distances, and different transmission modes such as reflection, diffraction and transmission.The final received signal is synthesized by the superposition of multipath signals with different phase and amplitude, and the phenomenon of fast fading will appear.Sharp changes can be observed when the relative distance of transceiver changes very little, so the fast changes in short distance are called small-scale fading. The propagation characteristics of wireless channel can be expressed by formula 6

$g=W \cdot Q \cdot j \quad(6)$

Where $\mathrm{W}$ is path loss, $\mathrm{q}$ is shadow fading and $\mathrm{j}$ is multipath fading.The specific value of each variable parameter can be calculated by formula 7 .

$$
\left\{\begin{array}{l}
W=10 \log 10\left[\frac{W_{t}}{W_{r}}\right] \\
Q=W\left(d_{0}\right)+10 n \log 10\left(\frac{d}{d_{0}}\right)+X_{\sigma}(t) \\
j(t, \tau)=\sum_{l=0}^{L} a_{l}(t) e^{-b 2 \pi f_{D}(t) t} \delta\left(\tau-\tau_{l}(t)\right)
\end{array}\right.
$$

Where $W_{t}$ and $W_{r}$ represent transmitting signal power and receiving signal power respectively. $d_{0}$ is the reference distance, $W\left(d_{0}\right)$ is the path loss at $d_{0}, \mathrm{n}$ is the path loss index, which is determined by the propagation environment. $X_{\sigma}(t)$ is the Gaussian random variable with the mean value of zero and the standard deviation of $\sigma$.In addition, $t$ and $\tau$ represent time and delay respectively, and $\mathrm{L}$ is the number of resolvable multipaths. $a_{l}(t)$ is the attenuation of each path, $\tau_{l}(t)$ is the propagation delay of each path, and $f_{D}(t)$ is the Doppler shift.By combining formula 6 with formula 7, we can get the result of the fading characteristic of the wireless communication channel with risk.

\subsection{Statistical information transmission risk probability}

Based on the channel fading characteristics, the risk value of wireless communication system channel information transmission is calculated, and the corresponding statistical results are obtained by means of probability statistics. The calculation process of channel transmission risk value is shown in Figure 8. 


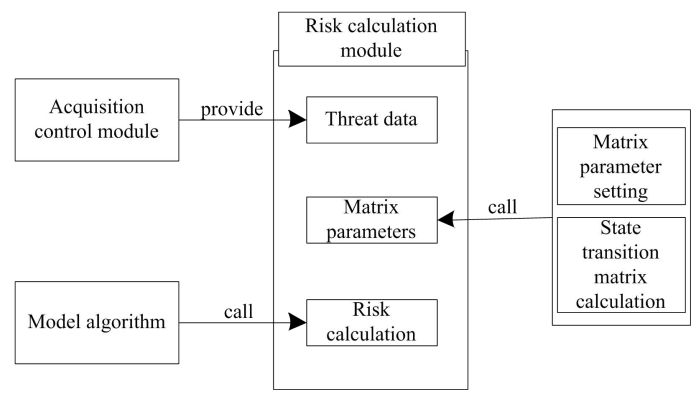

Fig. 8. Schematic diagram of risk calculation data processing

Call the data collection module to collect the original data, the intrusion detection system collects the external threat data in real time, the configuration verification system checks the internal configuration of the target host regularly, and returns the configuration results. The external threat data is the model observation value, the internal configuration is the internal threat data, and the internal and external cause correlation matrix is calculated with the external threat data.After the channel information data is obtained, the internal and external correlation matrix, observation value output matrix and initial state probability are called to calculate a new real-time state transition matrix according to the system changes caused by internal and external threats. Finally, the real-time risk total value of the target host is calculated based on the model algorithm combined with four probability matrices [10].Taking the interruption risk of large-scale wireless communication system channel as an example, if the channel remains constant during the transmission of the whole codeword, the interruption probability can be used to evaluate the system performance, and the corresponding interruption risk probability can be expressed as:

$$
P_{z}=P\left\{\log _{2}\left(1+\frac{\rho}{N} h^{H} V V^{H} h\right)<R\right\}
$$

By integrating all possible transmission risks, the statistical results of transmission risk probability can be obtained. The statistical method of channel information transmission risk probability in large-scale wireless communication system is realized.

\section{Experimental results and analysis}

In order to verify the statistical method of channel information transmission risk in large-scale wireless communication system, a performance comparison experiment is designed in the experimental environment. The experimental environment is a large-scale wireless communication system, which encodes the channel information in the wireless communication system. In this experiment, 12 channels are taken as the research object, and the statistical method of information transmission risk and the traditional statistical method are introduced 
into the background program of the system in the form of program code, which ensures the independent operation of the three statistical methods. The object of study is No.1-4 and No.5-8, respectively. The communication transmission channel is controlled by two traditional risk statistical methods, and the designed risk is controlled by two traditional risk statistical methods The statistical object of probability statistical method is the communication transmission channel numbered 8-12. The purpose of this experimental verification is divided into two parts. One is to verify the risk statistical error of the three methods by comparison, the other is to verify the application results of the three methods. The comparison results of risk statistical error of the three methods are shown in Figure 9.

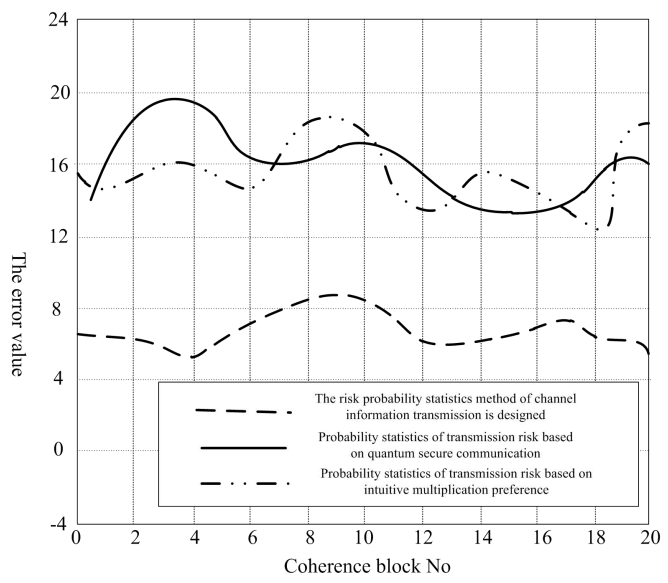

Fig. 9. Risk statistical error comparison curve

Figure 9 shows three cycles with 10 slots per cycle. The average error values of the two traditional risk statistical methods are 16.4 and 15.8 respectively, while the average error values of the channel information transmission risk statistical methods of the large-scale wireless communication system are 7.2 , which is reduced by about $56 \%$. Then, according to the results of the three risk statistical methods, the low risk value probability is selected to transmit the actual data information, and the stability of the system is observed according to the artificial adjustment of the external environment signal-to-noise ratio Sexual change, as shown in Table 2.

Table 2 Comparison results of wireless communication system stability

\begin{tabular}{|c|c|c|c|c|}
\hline $\begin{array}{l}\text { Signal-to-nois } \\
\text { e ratio }\end{array}$ & $\begin{array}{l}\text { Information } \\
\text { transmission } \\
\text { size/MB }\end{array}$ & $\begin{array}{c}\text { Probability } \\
\text { statistics of } \\
\text { transmission risk } \\
\text { based on } \\
\text { quantum secure }\end{array}$ & $\begin{array}{c}\text { Probability } \\
\text { statistics of } \\
\text { transmission risk } \\
\text { based on intuitive } \\
\text { multiplication }\end{array}$ & $\begin{array}{c}\text { Data reception } \\
\text { under the control } \\
\text { of design risk } \\
\text { probability } \\
\text { statistical method }\end{array}$ \\
\hline
\end{tabular}




\begin{tabular}{ccccc}
\hline & & communication & preference & /MB \\
\hline 1 & 4.31 & 3.58 & 3.42 & 3.88 \\
2 & 14.23 & 11.26 & 12.55 & 13.54 \\
3 & 4.08 & 2.34 & 2.78 & 3.27 \\
4 & 5.25 & 3.22 & 3.05 & 4.09 \\
5 & 8.70 & 7.25 & 7.12 & 8.10 \\
6 & 9.32 & 8.74 & 8.58 & 9.14 \\
7 & 3.79 & 2.08 & 1.97 & 3.31 \\
8 & 5.28 & 3.99 & 3.66 & 5.01 \\
9 & 11.23 & 10.81 & 10.67 & 11.08 \\
10 & 18.34 & 16.53 & 16.85 & 17.94 \\
\hline
\end{tabular}

In the calculation experiment, the actual data transmission volume is $84.53 \mathrm{mb}$, among which the actual data reception volume based on the transmission risk probability of quantum secure communication is $69.8 \mathrm{mb}$, the packet loss rate is $17.42 \%$, and the actual data reception volume based on the transmission risk probability of intuitive multiplication is $70.65 \mathrm{mb}$, The packet loss rate is $18.65 \%$, but in the design of large-scale wireless communication system, under the constraint of channel information transmission risk statistical method, the actual received data is $79.36 \mathrm{mb}$, that is to say, compared with the traditional method, the risk probability statistical method can effectively reduce the packet loss rate by about $11.31 \%$. Even in the case of serious noise interference, it can accurately select the channel with low risk probability for data transmission, which has high application value.

\section{Conclusion}

By collecting and counting the transmission state information of large-scale wireless communication system channel, this paper puts forward the statistical method of information transmission risk probability from the perspective of interference probability.The application value of the risk probability statistical method in the actual system operation is proved by the experimental verification. However, when the environment interference is large, there will still be some packet loss in the application of the method, which needs to be further optimized in the future research work.

\section{References}

[1] Boris Karanov, Tianhua Xu, Nikita A. Shevchenko, et al. Span length and information rate optimisation in optical transmission systems using single-channel digital backpropagation[J]. Optics Express, 2017, 25(21):25353. 
[2]Cho, Sungryung, Lee, Kyungrak, Kang, BooJoong,et al.: A hybrid MAC protocol for optimal channel allocation in large-scale wireless powered communication networks[J]. Eurasip Journal on Wireless Communications \& Networking, 2018,12(1):9-9

[3] Fatang Chen, Kang Yang, Pingping Xing,et al.: Multiuser pre-coding aided quadrature spatial modulation for large-scale MIMO channels[J]. Wireless Communication over ZigBee for Automotive Inclination Measurement. China Communications, 2018, 15(11):62-69

[4] Behrooz Makki, Tommy Svensson, Koen Buisman, et al.: Wireless Energy and Information Transmission in FSO and RF-FSO Links[J]. IEEE Wireless Communications Letters, 2017, 8(3):14-21

[5] Pilatte, Nicolas, Aristidou, Petros, Hug, Gabriela.: TDNetGen: An open-source, parametrizable, large-scale, transmission and distribution test system[J]. IEEE Systems Journal, 2017, 18(7):16-28

[6]David Elkouss, David Pérez-García.: Memory effects can make the transmission capability of a communication channel uncomputable[J]. Nature Communications, 2018, 9(1):1149

[7]V. L. Kolpashchikov.: Fluctuation-Dissipation Model of the Optical Information Transmission System[J]. Journal of Engineering Physics \& Thermophysics, 2018, 91(1):260-263

[8] A. I. Glazov, V. V. Grigor'ev, V. E. Kravtsov,et al.: International Comparisons of Standards in the Area of Fiber-Optic Communication and Information Transmission Systems[J]. Measurement Techniques, 2018, 60(10):1-7

[9]Hoang-Sy Nguyen, Thanh-Sang Nguyen, Miroslav Voznak.: Successful transmission probability of cognitive device-to-device communications underlaying cellular networks in the presence of hardware impairments[J]. Eurasip Journal on Wireless Communications \& Networking, 2017, 2017(1):208-208

[10] Lei Wang, Xu-Hui Xia, Jian-Hua Cao,et al.: Improved Ant Colony-Genetic Algorithm for Information Transmission Path Optimization in Remanufacturing Service System[J]. Chinese Journal of Mechanical Engineering, 2018, 31(1):107-107 